\title{
Classes of Unique Face and Its Classification
}

\author{
Mr.Raghavendra Kulkarni ${ }^{1}$, Dr. P. Nageswar Rao ${ }^{2}$ \\ ${ }^{1}$ Associate Prof. Dept.of ECE K.M.C.E\&T, Devarkonda under JNT University, Hyderabad, A.P. \\ raghavendrakulkarni444@gmail.com \\ ${ }^{2}$ Principal K.M.C.E\&T, Devarkonda, under JNT University, Hyderabad, A. P. \\ pnrao33@gmail.com
}

\begin{abstract}
Near resembling faces ,Look alike faces, disguised faces and many more are today's challenges for researchers in the field of face recognition and these challenges become more serious in case of large facial Variations. Humans are able to identify reliably a large number of faces but a automated face recognition system must be face specific, it should effectively use features that discriminate a face from others by preferably amplifying distinctive characteristics of face. Face recognition has drawn wide attention from researchers in areas of machine learning, computer vision, pattern recognition, neural networks, access control, information security, law enforcement and surveillance, smart cards etc. This paper shows that how the unique face which is having a unique singular value per face under different variations is effectively classified and recognized.
\end{abstract}

\section{Indexing terms}

Unique face, DR, SV, SVD, PCA

\section{Council for Innovative Research}

Peer Review Research Publishing System

Journal: International Journal of Management \& Information Technology

Vol. 7, No. 1

editor@cirworld.com

www.cirworld.com, member.cirworld.com 


\section{INTRODUCTION}

Researchers started paying attention to the subject of face recognition from the year 1960 and the world of scholars attracted towards the diverse approaches from the year 1980. The first automated algorithmic approach was developed in the year 1973.Researchers concluded that humans have a specialized facial recognition mechanism which only focuses on particular areas or features for recognition, this conclusion the researchers applied for dimensionality reduction techniques. The introduction of abstract mathematical tools like eigenfaces created another approach to face recognition and ultimately given up a decomposition algorithm for calculating singular values by which in this paper a unique face and its classes have been defined ,tested and classified

\section{CHALLENGES OF FACE RECOGNITION}

The problem of face recognition is very challenging because faces are non-rigid and have a high degree of variability in size, shape, color and texture. More precisely the challenges associated with face detection are due to:

a) Pose: The images of a face vary due to the relative camera-face pose (frontal, 45 degree, profile, upside down),

b) and some facial features such as an eye or the nose may become partially or wholly occluded.

c) Presence or absence of structural components: Facial features such as beards, mustaches, and glasses may or may not be present and there is a great deal of variability among these components including shape, color, and size.

d) Facial expression: The appearances of faces are directly affected by a person's facial expression.

e) Occlusion: Faces may be partially occluded by other objects. In an image with a group of people, some faces may partially occlude other faces.

f) Image orientation: Face images directly vary for different rotations about the camera's optical axis.

g) Imaging conditions: When the image is formed, factors such as lighting (spectra, source distribution and intensity) and camera characteristics (sensor response, lenses) affect the appearance of a face.

\section{UNIQUE FACE RECOGNITION APPROACH}

The generic recognition system shown in figure 1 below:

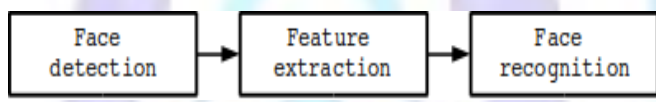

Figure 1: A generic face recognition system

The input of a face recognition system is always an image. The output is an identification or verification of the subject or subjects that appear in the image. The approach define a face recognition system as a three step process as shown in Figure 1.TheFace Detection and Feature Extraction phases could run simultaneously. Face detection identifies a certain image region as a face. The feature extraction step involves obtaining relevant facial features from the data.

These features could be certain face regions, variations, angles or measures, which can be human relevant. In this paper singular values (SV) are obtained as face features which are said to be unique and singular value which has highest value is said to be the first SV and it will be having large information with it. These SV'S are arranged in decreasing order of magnitude. Finally, the system does recognize the face. In an identification task, the system would report an identity from a database.

This phase involves a comparison method, a classification algorithm and an accuracy measure.

Every Image Matrix A can always have the SVDas

$$
A=U \Sigma V^{T}
$$

Where $\mathrm{U}$ is an $\mathrm{m} \times \mathrm{m}$ orthogonal matrix; $\mathrm{V}$ an $\mathrm{n} \times \mathrm{n}$ orthogonal matrix, and $\sum$ is an $\mathrm{m} \times \mathrm{n}$ matrix containing the singular values of $A$.

$$
\sigma_{1} \geq \sigma_{2} \geq \cdots \geq \sigma_{n} \geq 0
$$

along its main diagonal. These SVD features are used for facial feature decomposition to represent an image in dimensionality reduction (DR) factor. An SVD operation breaks down the matrix $A$ into three separate matrices.

$$
\begin{aligned}
& A=U \Sigma V^{T} \\
& =\left[u_{1}, \ldots u_{n}\right]\left[\begin{array}{llll}
\sigma_{1} & & \\
& & \ldots & \\
& & \sigma_{n}
\end{array}\right]\left[\begin{array}{l}
v_{1}^{T} \\
\vdots \\
v_{n}^{T}
\end{array}\right] \\
& =\left[u_{1}, \ldots u_{n}\right]\left[\begin{array}{c}
\sigma_{1} v_{1}^{T} \\
: \\
\sigma_{n} v_{n}^{T}
\end{array}\right] \\
& =\sigma_{1} u_{1} v_{1}^{T}+\ldots+\sigma_{n} u_{n} v_{n}^{T} \\
& =\sigma_{1} u_{1} v_{1}^{T}+\ldots+\sigma_{r} u_{r} v_{r}^{T}
\end{aligned}
$$


because $\sigma_{r+1} \ldots \sigma_{n}=$ are equal to zeros.For each face image matrix A which has the SVD, its modified SVD is defined as:

$$
M=U \Sigma^{\alpha} V^{\top}
$$

Where $U, \Sigma$ and $V$ are the SV matrices, and in order to alleviate the facial variations on face images a fractional parameter $\alpha$ is used which satisfies the condition:

$$
0 \leq \alpha \leq 1
$$

The main ideas are:

1) The weights of the leading base images $u_{i} v_{i}^{\top}$ should be deflated, since they are very sensitive to the great facial variations within the image matrix $A$ itself.

2) The weights of base images $u_{i} v_{i}^{\top}$ corresponding to relatively small $\sigma_{i}$ 's should be inflated, since they may be less sensitive to the facial variations within $A$.

3) The order of the weights of the base images $u_{i} v_{i}^{\top}$ in formulating the new representation of SVD should be retained.

4) The fractional parameter, a must inflates the lower SV.

Unique singular values are obtained by the following steps:

1) Apply SVD on each of the face image for each class in the data base to get singular vector.

2) The obtained Singular Vector is applied with the fractional value of aand a optimized SVD values are obtained .

3) Each training face image is then projected using the these obtained face feature image.

4) For the obtained representing image apply a DR method PCA, where the Eigen features are Computed and for the maximum Eigen values Eigen vectors are located and normalized for this Projected image.

5) A test face image is transformed into a face feature matrix.

6) For the developed query feature a image representation is developed and passed to the PCA.

7) For the computed face feature the distance between a test face image $T$ and a traning face imagesis Calculated.

8) Least distance will be the recognized image.

\section{CLASSIFICATION AND ANALYSIS}

The steps \& effects are illustrated and presented below by considering the classes of unique face.Some of the self database images and query images (set consists of color, black \& white images) with different classes considered are:

\section{Query Images}
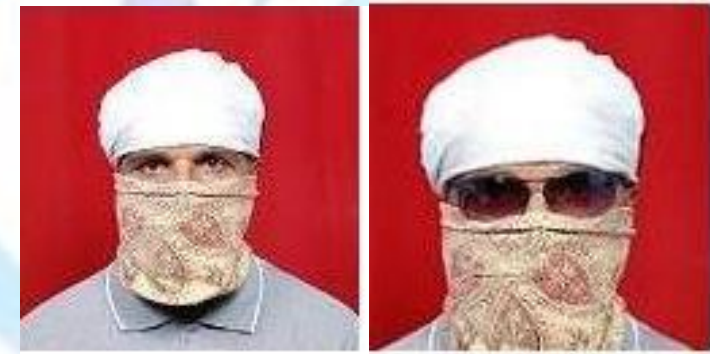

Fig.2: sample query images considered

\section{Database Images}

For the implementation of the proposed face recognition architecture the database samples are trained for the knowledge creation for classification. During training phase when a new facial image is added to the system the features are calculated and aligned for the dataset formation. These data sets consist of the image index and its corresponding features extracted. This feature table is created for the entire database image and passed for recognition.
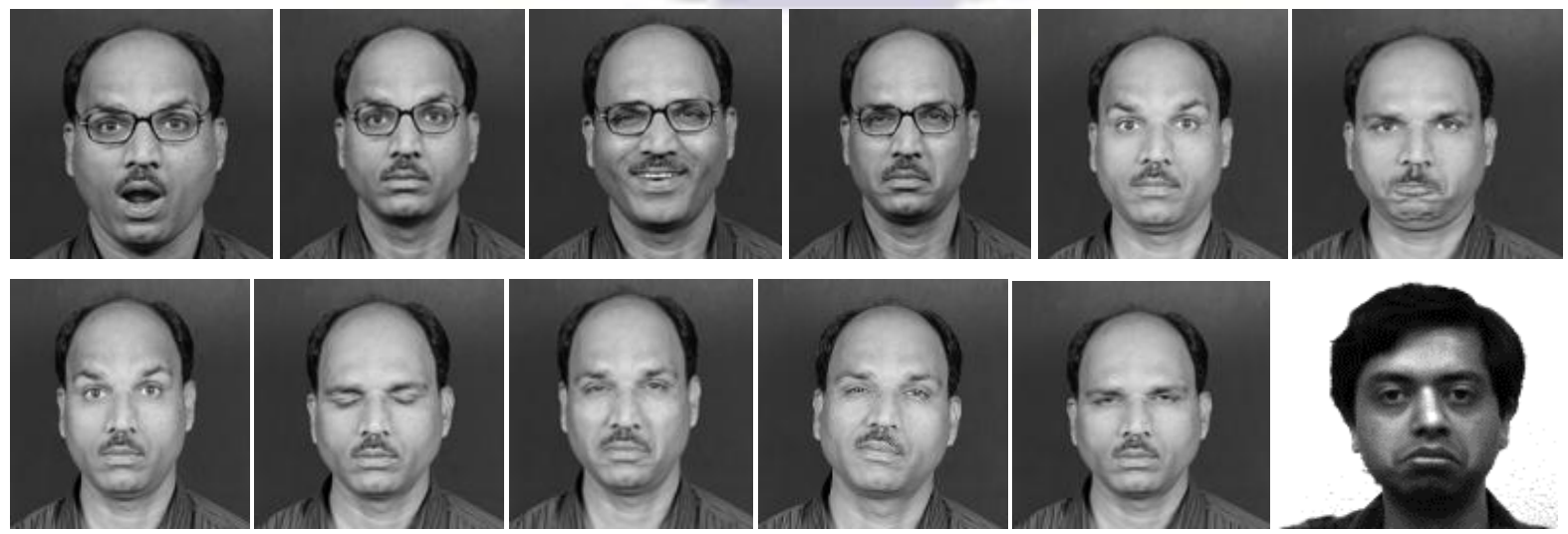

Figure 3:shows typical database images considered for face recognition 
A) CLASS 1:Image with No variation
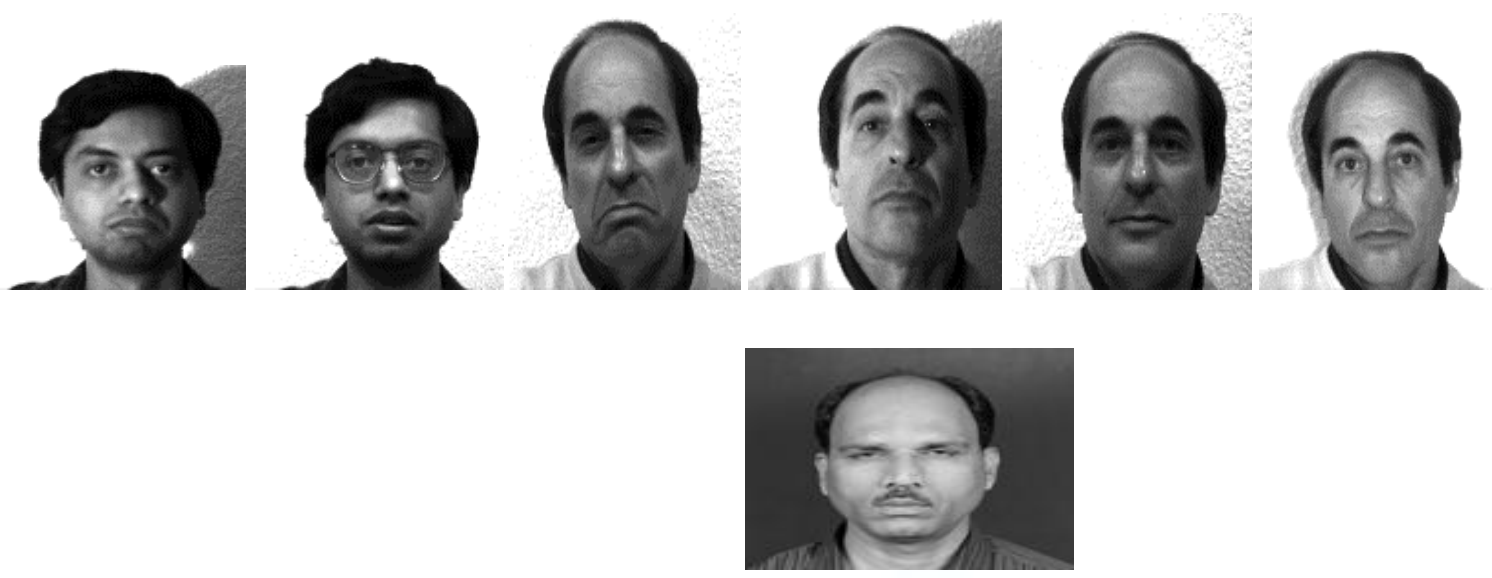

Original query image

Fig. 4: Original query image

Output with $\alpha=1$

Output with $\alpha=0.5$

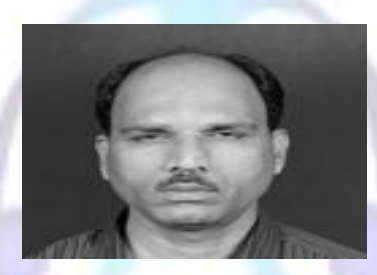

Recognized image

Fig 5: obtained results with $\alpha=1$
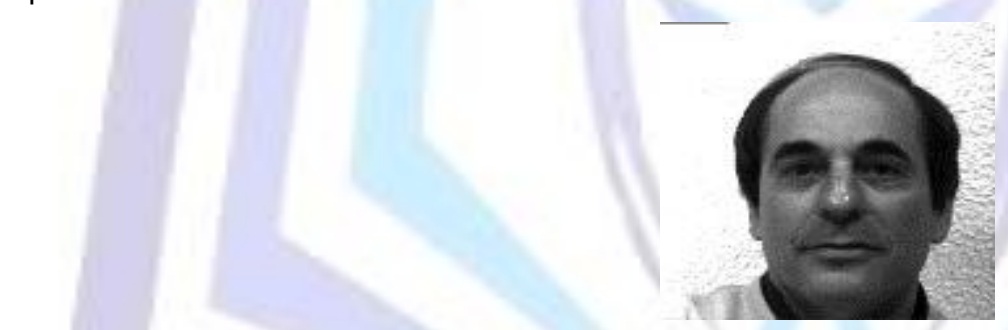

Fig 6: obtained result with $\alpha=0.5$

\section{B) CLASS 2:Image having occlusions}

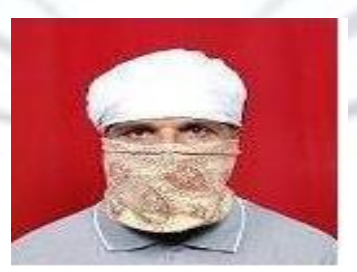

Fig 7: original query image

Output with $\alpha=1$

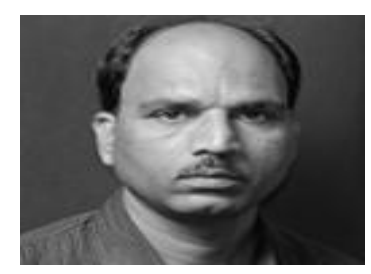

Fig 8: obtained result with $\alpha=1$ 
C) CLASS 3:Image having occlusions

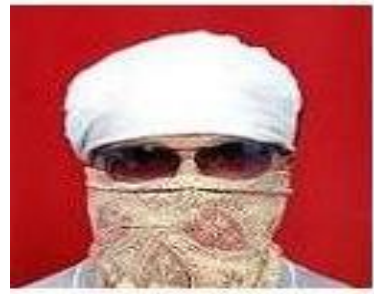

Fig 9: original query image

Output with $\alpha=1$

\section{CONCLUSION}

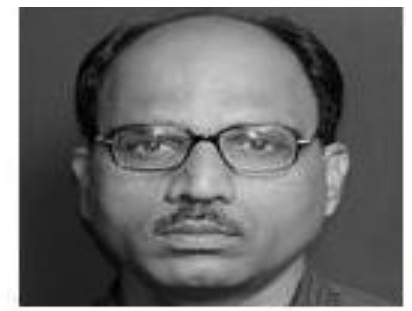

Fig 10: obtained result with $\alpha=1$

In olden days for investigation security people used to take help of face sketches to be identified by a human, which implies that humans do store the specific face features for classification as face or no face. The same method is applied for machines to be face specific. In my previous published paper a preliminary study was considered but in this paper classes of my own faces are presented and are effectively classified.The algorithm used can result in higher accuracy in estimation as compared to the previous techniques for face recognition. It is presented that the faces of different subjects are classified based on unique amplification factor $\alpha$, if different value of $\alpha$ is used it can be seen from case 1 that a no face results. As most research works are happening towards the recognition of facial images in both static information and continuous information, this method could be very suitable for continuous environment due to large observed external effects.

\section{REFERENCES}

[1] M. Turk, A. Pentland, Eigenfaces for recognition, J. Cognitive Neurosci. 3 (1) (1991) 71-96.

[2] J. Liu, S. Chen, Non-iterative generalized low rank approximation of matrices, Pattern Recognition Lett. 27 (9) (2006) 1002-1008.

[3] P. Belhumeur, J. Hespanha, D.J. Kriegman, Eigenfaces vs. Fisherfaces:recognition using class specific linear projection, IEEE Trans. Pattern Anal. Mach. Intell. 19 (7) (1997) 711-720.

[4] J. Liu, S. Chen, Resampling Ida/qr and pca+lda for face recognition, in: The 18th Australian Joint Conference on Artificial Intelligence, 2005, pp. 1221-1224.

[5] Z. Hong, Algebraic feature extraction of image for recognition, Pattern Recognition 24 (1991) 211-219.

[6] Y. Cheng, K. Liu, J. Yang, Y. Zhuang, N. Gu, Human face recognition method based on the statistical model of small sample size, in: Intelligent Robots and Computer Vision X: Algorithms and Techniques, 1991, pp. 85-95.

[7] Y. Tian, T. Tan, Y. Wang, Y. Fang, Do singular values contain adequate information for face recognition, Pattern Recognition 36 (3) (2003) 649-655.

[8] Jun Liu, Songcan Chen*, Xiaoyang Tan ,Fractional order singular value decomposition representation for face recognition,Pattern Recognition 41 (2008) 378 - 395

\section{Authors Profiles}

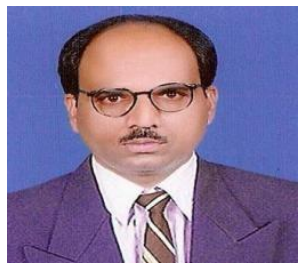

Raghavendra Kulkarni Working as Associate Professor in ECE department, Khader Memorial College Engg. \& Technology. Devarkonda Nalgonda (Dist), Andhra Pradesh, India. He received B. Tech. (ECE) from Gulbarga University, M. Tech Degree in DSCE from JNTU Hyderabad. He is pursuing Ph.D from MEWAR UNIVERSAITY,RAJASTHAN.He is having 21 years of experience. He has authored and coauthored 45 research papers in National and International Conferences \& Jourals. 


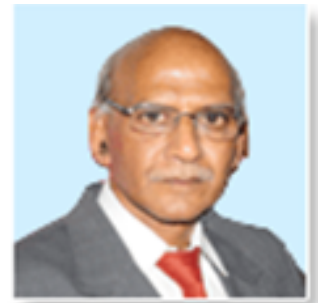

Dr. P. Nageshwar Rao Working as Principal at Khader Memorial College of Engg. \& Technology, Devarkonda, Nalgondea (Dist). Andhra Pradesh, India. He received B.E from Bangalore University, M.Tech form Andhra University \& Ph.D. form Nagarjuna University .He Published nearly 10 papers and guided six scholars and his Research area is on Data Transmission in real time environment. 